\title{
Multiple positive solutions for mixed fractional differential system with $p$-Laplacian operators
}

Ying Wang ${ }^{1 *}$

*Correspondence: lywy1981@163.com 'School of Mathematics and Statistics, Linyi University, Linyi, People's Republic of China

\section{Springer}

\begin{abstract}
This paper is focused on researching a class of mixed fractional differential system with $p$-Laplacian operators. Based on the properties of the corresponding Green's function, different combinations of superlinearity or sublinearity for the nonlinearities and other appropriate conditions, the existence of multiple positive solutions are derived via the Guo-Krasnosel'skii fixed point theorem. An example is then given to illustrate the usability of the main results.
\end{abstract}

MSC: 26A33; 34B18

Keywords: Multiple positive solutions; Mixed fractional differential system; $p$-Laplacian operators; Coupled integral boundary conditions

\section{Introduction}

In this paper, we investigate the following mixed fractional differential system:

$$
\left\{\begin{array}{l}
\left.D^{\beta_{1}}\left(\varphi_{p_{1}}{ }^{c} D^{\alpha_{1}} u(t)\right)\right)+f_{1}(t, u(t), v(t))=0, \\
D^{\beta_{2}}\left(\varphi_{p_{2}}\left({ }^{c} D^{\alpha_{2}} v(t)\right)\right)+f_{2}(t, u(t), v(t))=0, \quad 0<t<1, \\
u^{\prime}(0)=u^{\prime \prime}(0)=\cdots=u^{(n-1)}(0)=0, \quad u(1)=\mu_{1} \int_{0}^{1} a(s) v(s) d A_{1}(s), \\
v^{\prime}(0)=v^{\prime \prime}(0)=\cdots=v^{(m-1)}(0)=0, \quad v(1)=\mu_{2} \int_{0}^{1} b(s) u(s) d A_{2}(s), \\
{ }^{c} D^{\alpha_{1}} u(0)=0, \quad{ }^{c} D^{\alpha_{1}} u(1)=\varepsilon_{1}{ }^{c} D^{\alpha_{1}} u\left(\eta_{1}\right), \\
{ }^{c} D^{\alpha_{2}} v(0)=0, \quad{ }^{c} D^{\alpha_{2}} v(1)=\varepsilon_{2}{ }^{c} D^{\alpha_{2}} v\left(\eta_{2}\right),
\end{array}\right.
$$

where $1<\beta_{i} \leq 2,1 \leq n-1<\alpha_{1} \leq n, 1 \leq m-1<\alpha_{2} \leq m, n, m \geq 2, D^{\beta_{i}}$ is the RiemannLiouville derivative operator, ${ }^{c} D^{\alpha_{i}}$ is the Caputo derivative. $\mu_{i}>0$ is a constant, $\eta_{i} \in(0,1)$, $\varepsilon_{i}>0$ and satisfies $1-\varepsilon_{i}^{p_{i}-1} \eta^{\beta_{i}-1}>0, \varphi_{p_{i}}$ is the Laplacian operator defined by $\varphi_{p_{i}}(s)=|s|^{p_{i}-2} s$, $\left(\varphi_{p_{i}}\right)^{-1}=\varphi_{q_{i}}, \frac{1}{p_{i}}+\frac{1}{q_{i}}=1, p_{i}>1, \int_{0}^{1} a(s) v(s) d A_{1}(s), \int_{0}^{1} b(s) u(s) d A_{2}(s)$ denote the RiemannStieltjes integrals with a signed measure, that is $A_{i}:[0,1] \rightarrow[0,+\infty)$ is the function of bounded variation. $a, b:[0,1] \rightarrow[0,+\infty)$ are continuous, $f_{i}:[0,1] \times[0,+\infty) \times[0,+\infty) \rightarrow$ $[0,+\infty)$ is a continuous function, $i=1,2$.

Compared with the integer order systems, fractional differential systems are regarded as a better tool in the description of some problems in science and engineering. Arafal et

(c) The Author(s) 2019. This article is distributed under the terms of the Creative Commons Attribution 4.0 International License (http://creativecommons.org/licenses/by/4.0/), which permits unrestricted use, distribution, and reproduction in any medium, provided you give appropriate credit to the original author(s) and the source, provide a link to the Creative Commons license, and indicate if changes were made. 
al. [1] presented a fractional order model for infection of $\mathrm{CD} 4^{+} \mathrm{T}$ cells:

$$
\left\{\begin{array}{l}
D^{\alpha_{1}}(T)=s-K V T-d T+b I \\
D^{\alpha_{2}}(I)=K V T-(b+\delta) I \\
D^{\alpha_{3}}(V)=N \delta I-c V
\end{array}\right.
$$

where $\alpha_{1}, \alpha_{2}, \alpha_{3}>0$. In the mathematical context, many mathematicians and applied scholars have studied the fractional differential equation or system in recent years [2-15]. In addition, by applying the functional analysis methods such as the lower and upper solutions, monotone iterative techniques, fractional integro-differential equations or singular equations are researched by Dumitru et al. [16], Denton et al. [17], Lyons and Neugebauer [18], Ambrosio [19], Zhou and Qiao [20]. There are also related books [21, 22].

Cabada and Wang in [23] studied the following factional differential equation:

$$
\left\{\begin{array}{l}
D^{\alpha} u(t)+f(t, u(t))=0, \quad 0<t<1, \\
u(1)=u^{\prime}(0)=0, \quad u(1)=\lambda \int_{0}^{1} u(s) d A(s),
\end{array}\right.
$$

where $2<\alpha \leq 3,0<\lambda, \lambda \neq \alpha, D^{\alpha}$ is the Caputo fractional derivative, and $f:[0,1] \times$ $[0,+\infty) \rightarrow[0,+\infty)$ is a continuous function. By the use of Guo-Krasnosel'skii fixed point theorem, the authors in [23] obtained the positive solution to Eq. (1.2). Cabada and Wang also discussed the solution of Eq. (1.2) when $D^{\alpha}$ is the Riemann-Liouville fractional derivative [24].

The $p$-Laplacian equation is the second order quasilinear differential operator, it arises in the modeling of various physical and natural phenomena. Fractional differential equation with $p$-Laplacian operator can describe the nonlinear phenomena in non-Newtonian fluids and establishes complex process models; for some related articles, see [25-31]. Via variational methods, Li and Wei [32] dealt with fractional $p$-Laplacian equations, the existence and multiplicity of nontrivial solutions were obtained. Wu et al. [33] researched the following fractional differential turbulent flow model and obtained the iterative solutions of the equation:

$$
\left\{\begin{array}{l}
-D^{\alpha}\left(\varphi_{p}\left(-D^{\gamma} u(t)\right)\right)=g(t) h(u), \quad 0<t<1, \\
u(0)=0, \quad D^{\gamma} u(0)=D^{\gamma} u(1)=0, \quad u(1)=\int_{0}^{1} u(s) d A(s),
\end{array}\right.
$$

where $1<\alpha, \gamma \leq 2, D^{\alpha}, D^{\gamma}$ are the Riemann-Liouville fractional derivatives, $h:[0,+\infty) \rightarrow$ $[0,+\infty)$ is a continuous and increasing function.

Fractional differential systems with $p$-Laplacian operators have also attracted tremendous attention [34-40]. Among them, applying the monotone iterative approach, the authors in [34] got the extremal solutions of the following system:

$$
\left\{\begin{array}{l}
D_{0^{+}}^{\alpha_{1}}\left(\varphi_{p_{1}}\left(D_{0^{+}}^{\beta_{1}} u(t)\right)\right)=f_{1}(t, v(t)), \\
D_{0^{+}}^{\alpha_{2}}\left(\varphi_{p_{2}}\left(D_{0^{+}}^{\beta_{2}} v(t)\right)\right)=f_{2}(t, u(t)), \quad 0<t<1, \\
u(0)=D_{0^{+}}^{\beta_{1}} u(0)=0, \quad D_{0^{+}}^{\gamma_{1}} u(1)=\sum_{j=1}^{m-2} a_{1 j} D_{0^{+}}^{\gamma_{1}} u\left(\eta_{j}\right)=0, \\
v(0)=D_{0^{+}}^{\beta_{2}} v(0)=0, \quad D_{0^{+}}^{\gamma_{2}} v(1)=\sum_{j=1}^{m-2} a_{2 j} D_{0^{+}}^{\gamma_{2}} v\left(\eta_{j}\right)=0,
\end{array}\right.
$$


where $0<\alpha_{i}, \gamma_{i} \leq 1,1<\beta_{i} \leq 2, D_{0^{+}}^{\alpha_{i}}, D_{0^{+}}^{\beta_{i}} D_{0^{+}}^{\gamma_{i}}$ are the Riemann-Liouville fractional derivatives, $i=1,2$.

Inspired by the above articles, in this article we discuss the mixed fractional differential system with $p$-Laplacian operators under integral boundary value conditions. To the best of our knowledge, there is very little research on mixed fractional differential systems, especially if the system has $p$-Laplacian operators. Through the application of the Guo-Krasnosel'skii fixed point theorem, the existence of multiple positive solutions of the system is achieved.

\section{Preliminaries and lemmas}

Definition 2.1 $([41,42])$ The Caputo fractional order derivative of order $\alpha>0, n-1<\alpha<$ $n, n \in \mathbb{N}$ is defined as

$$
{ }^{c} D^{\alpha} u(t)=\frac{1}{\Gamma(n-\alpha)} \int_{0}^{t}(t-s)^{n-\alpha-1} u^{(n)}(s) d s,
$$

where $u \in C^{n}(J, \mathbb{R}), \mathbb{R}=(-\infty,+\infty), \mathbb{N}$ denotes the natural number set, $n=[\alpha]+1$, and $[\alpha]$ denotes the integer part of $\alpha$.

Definition $2.2([41,42])$ Let $\alpha>0$ and let $u$ be piecewise continuous on $(0,+\infty)$ and integrable on any finite subinterval of $[0,+\infty)$. Then, for $t>0$, we call

$$
I^{\alpha} u(t)=\frac{1}{\Gamma(\alpha)} \int_{0}^{t}(t-s)^{\alpha-1} u(s) d s
$$

the Riemann-Liouville fractional integral of $u$ of order $\alpha$.

Lemma $2.1([41,42])$ Let $n-1<\alpha \leq n, u \in C^{n}[0,1]$. Then

$$
I^{\alpha}\left({ }^{c} D^{\alpha} u\right)(t)=u(t)+c_{0}+c_{1} t+c_{2} t^{2}+\cdots+c_{n-1} t^{n-1},
$$

where $c_{i} \in \mathbb{R}(i=1,2, \ldots, n-1), n$ is the smallest integer greater than or equal to $\alpha$.

Let $\left.\varphi_{p_{1}}{ }^{(} D_{0^{+}}^{\alpha_{1}} u(t)\right)=\bar{u}(t), \varphi_{p_{2}}\left({ }^{c} D_{0^{+}}^{\alpha_{2}} v(t)\right)=\bar{v}(t)$, then $\bar{u}(0)=0, \bar{u}(1)=\varepsilon_{1}^{p_{1}-1} \bar{u}\left(\eta_{1}\right), \bar{v}(0)=0$, $\bar{v}(1)=\varepsilon_{2}^{p_{2}-1} \bar{v}\left(\eta_{2}\right)$, we now consider the following system:

$$
\left\{\begin{array}{l}
D^{\beta_{1}} \bar{u}(t)+y_{1}(t)=0, \quad D^{\beta_{2}} \bar{v}(t)+y_{2}(t)=0, \quad 0<t<1, \\
\bar{u}(0)=\bar{v}(0)=0, \quad \bar{u}(1)=\varepsilon_{1}^{p_{1}-1} u\left(\eta_{1}\right), \\
\bar{v}(1)=\varepsilon_{2}^{p_{2}-1} v\left(\eta_{2}\right) .
\end{array}\right.
$$

Similar to [43], if $y_{i} \in C[0,1]$, then the system (2.1) has a unique solution,

$$
\left\{\begin{array}{l}
\bar{u}(t)=\int_{0}^{1} \bar{H}_{1}(t, s) y_{1}(s) d s, \\
\bar{v}(t)=\int_{0}^{1} \bar{H}_{2}(t, s) y_{2}(s) d s
\end{array}\right.
$$


where

$$
\begin{aligned}
& \bar{H}_{i}(t, s)=\bar{h}_{i}(t, s)+\frac{\varepsilon_{i}^{p_{i}-1} t^{\beta_{i}-1}}{1-\varepsilon_{i}^{p_{i}-1} \eta_{i}^{\beta_{i}-1}}, \\
& \bar{h}_{i}(t, s)= \begin{cases}\frac{(t(1-s))^{\beta_{i}-1}-(t-s){ }^{\beta_{i}-1}}{\Gamma\left(\beta_{i}\right)}, & 0 \leq s \leq t \leq 1, \\
\frac{\left(t(1-s) \beta_{i}-1\right.}{\Gamma\left(\beta_{i}\right)}, & 0 \leq t \leq s \leq 1 .\end{cases}
\end{aligned}
$$

For $y_{i} \in C[0,1]$, consider the system

$$
\left\{\begin{array}{l}
D^{\beta_{1}}\left(\varphi_{p_{1}}\left({ }^{c} D^{\alpha_{1}} u(t)\right)\right)+y_{1}(t)=0, \quad D^{\beta_{2}}\left(\varphi_{p_{2}}\left({ }^{c} D^{\alpha_{2}} v(t)\right)\right)+y_{2}(t)=0, \quad 0<t<1, \\
u^{\prime}(0)=u^{\prime \prime}(0)=\cdots=u^{(n-1)}(0)=0, \quad u(1)=\mu_{1} \int_{0}^{1} a(s) v(s) d A_{1}(s), \\
v^{\prime}(0)=v^{\prime \prime}(0)=\cdots=v^{(m-1)}(0)=0, \quad v(1)=\mu_{2} \int_{0}^{1} b(s) u(s) d A_{2}(s), \\
{ }^{c} D^{\alpha_{1}} u(0)=0, \quad{ }^{c} D^{\alpha_{1}} u(1)=\varepsilon_{1}{ }^{c} D^{\alpha_{1}} u\left(\eta_{1}\right), \\
{ }^{c} D^{\alpha_{2}} v(0)=0, \quad{ }^{c} D^{\alpha_{2}} v(1)=\varepsilon_{2}{ }^{c} D^{\alpha_{2}} v\left(\eta_{2}\right) .
\end{array}\right.
$$

Through calculation, we conclude that system (2.3) is equal to

$$
\begin{cases}{ }^{c} D^{\alpha_{1}} u(t)+\varphi_{q_{1}}\left(\int_{0}^{1} \bar{H}_{1}(t, s) y_{1}(s) d s\right)=0, & \\ { }^{c} D^{\alpha_{2}} v(t)+\varphi_{q_{2}}\left(\int_{0}^{1} \bar{H}_{2}(t, s) y_{2}(s) d s\right)=0, & 0<t<1, \\ u^{\prime}(0)=u^{\prime \prime}(0)=\cdots=u^{(n-1)}(0)=0, & u(1)=\mu_{1} \int_{0}^{1} a(s) v(s) d A_{1}(s), \\ v^{\prime}(0)=v^{\prime \prime}(0)=\cdots=v^{(m-1)}(0)=0, & v(1)=\mu_{2} \int_{0}^{1} b(s) u(s) d A_{2}(s) .\end{cases}
$$

Lemma 2.2 was obtained by the author herself and her collaborator in [44]

Lemma 2.2 Assume the following condition $\left(\mathbf{H}_{0}\right)$ holds.

$\left(\mathbf{H}_{0}\right)$

$$
k_{1}=\int_{0}^{1} a(s) d A_{1}(s)>0, \quad k_{2}=\int_{0}^{1} b(s) d A_{2}(s)>0, \quad 1-\mu_{1} \mu_{2} k_{1} k_{2}>0 .
$$

Let $h_{i} \in C(0,1) \cap L(0,1)(i=1,2)$, then the system with the coupled boundary conditions

$$
\begin{cases}{ }^{c} D^{\alpha_{1}} u(t)+h_{1}(t)=0, \quad{ }^{c} D^{\alpha_{2}} v(t)+h_{2}(t)=0, & 0<t<1, \\ u^{\prime}(0)=u^{\prime \prime}(0)=\cdots=u^{(n-1)}(0)=0, & u(1)=\mu_{1} \int_{0}^{1} a(s) v(s) d A_{1}(s), \\ v^{\prime}(0)=v^{\prime \prime}(0)=\cdots=v^{(m-1)}(0)=0, & v(1)=\mu_{2} \int_{0}^{1} b(s) u(s) d A_{2}(s),\end{cases}
$$

has a unique integral representation,

$$
\left\{\begin{array}{l}
u(t)=\int_{0}^{1} K_{1}(t, s) h_{1}(s) d s+\int_{0}^{1} H_{1}(t, s) h_{2}(s) d s \\
v(t)=\int_{0}^{1} K_{2}(t, s) h_{2}(s) d s+\int_{0}^{1} H_{2}(t, s) h_{1}(s) d s
\end{array}\right.
$$


where

$$
\begin{aligned}
& K_{1}(t, s)=\frac{\mu_{1} \mu_{2} k_{1}}{1-\mu_{1} \mu_{2} k_{1} k_{2}} \int_{0}^{1} G_{1}(t, s) b(t) d A_{2}(t)+G_{1}(t, s), \\
& H_{1}(t, s)=\frac{\mu_{1}}{1-\mu_{1} \mu_{2} k_{1} k_{2}} \int_{0}^{1} G_{2}(t, s) a(t) d A_{1}(t), \\
& K_{2}(t, s)=\frac{\mu_{2} \mu_{1} k_{2}}{1-\mu_{1} \mu_{2} k_{1} k_{2}} \int_{0}^{1} G_{2}(t, s) a(t) d A_{1}(t)+G_{2}(t, s), \\
& H_{2}(t, s)=\frac{\mu_{2}}{1-\mu_{1} \mu_{2} k_{1} k_{2}} \int_{0}^{1} G_{1}(t, s) b(t) d A_{2}(t),
\end{aligned}
$$

and

$$
G_{i}(t, s)= \begin{cases}\frac{(1-s)^{\alpha_{i}-1}-(t-s)^{\alpha_{i}-1}}{\Gamma\left(\alpha_{i}\right)}, & 0 \leq s \leq t \leq 1, \quad i=1,2 . \\ \frac{(1-s)^{\alpha_{i}-1}}{\Gamma\left(\alpha_{i}\right)}, & 0 \leq t \leq s \leq 1,\end{cases}
$$

Lemma 2.3 The Green function $\bar{H}_{i}(t, s), G_{i}(t, s)(i=1,2)$ defined separately by (2.2), (2.7) has the following properties:

(i) $\bar{H}_{i}(t, s), G_{i}(t, s):[0,1] \times[0,1] \rightarrow[0,+\infty)$ are continuous,

(ii)

$$
\frac{(1-s)^{\alpha_{i}-1}\left(1-t^{\alpha_{i}-1}\right)}{\Gamma\left(\alpha_{i}\right)} \leq G_{i}(t, s) \leq \frac{(1-s)^{\alpha_{i}-1}}{\Gamma\left(\alpha_{i}\right)}, \quad t, s \in[0,1]
$$

Proof Obviously, (i) holds, we only prove (ii). From the definition of $G_{i}(t, s)$, for $0 \leq t \leq$ $s \leq 1$, it is obvious that (ii) holds.

For $0 \leq s \leq t \leq 1$, we have $t-t s \geq t-s$, then

$$
\begin{aligned}
(1-s)^{\alpha_{i}-1}-(t-s)^{\alpha_{i}-1} & \geq(1-s)^{\alpha_{i}-1}-(t-t s)^{\alpha_{i}-1} \\
& \geq(1-s)^{\alpha_{i}-1}-t^{\alpha_{i}-1}(1-s)^{\alpha_{i}-1} \\
& =(1-s)^{\alpha_{i}-1}\left(1-t^{\alpha_{i}-1}\right),
\end{aligned}
$$

so, we know $\frac{(1-s)^{\alpha_{i}-1}\left(1-t^{\alpha_{i}-1}\right)}{\Gamma\left(\alpha_{i}\right)} \leq G_{i}(t, s)$. It is also defined by $G_{i}(t, s)$, and we obtain $G_{i}(t, s) \leq$ $\frac{(1-s)^{\alpha_{i}}}{\Gamma\left(\alpha_{i}\right)}$. Thus, (ii) holds. The proof is completed.

Similar to the proof in [35], Lemma 2.4 was obtained.

Lemma 2.4 For $t, s \in[0,1]$, the functions $K_{i}(t, s)$ and $H_{i}(t, s)(i=1,2)$ defined as $(2.3)$ satisfy

$$
\begin{array}{ll}
K_{1}(t, s), H_{2}(t, s) \leq \rho(1-s)^{\alpha_{1}-1}, & K_{2}(t, s), H_{1}(t, s) \leq \rho(1-s)^{\alpha_{2}-1}, \\
K_{1}(t, s), H_{2}(t, s) \geq \varrho(1-s)^{\alpha_{1}-1}, & K_{2}(t, s), H_{1}(t, s) \geq \varrho(1-s)^{\alpha_{2}-1},
\end{array}
$$


where

$$
\begin{aligned}
& \rho=\max \left\{\begin{array}{l}
\frac{\mu_{1} \mu_{2} k_{1}}{\Gamma\left(\alpha_{1}\right)\left(1-\mu_{1} \mu_{2} k_{1} k_{2}\right)} \int_{0}^{1} b(t) d A_{2}(t)+\frac{1}{\Gamma\left(\alpha_{1}\right)}, \frac{\mu_{2}}{\Gamma\left(\alpha_{1}\right)\left(1-\mu_{1} \mu_{2} k_{1} k_{2}\right)} \int_{0}^{1} b(t) d A_{2}(t), \\
\frac{\mu_{1} \mu_{2} k_{2}}{\Gamma\left(\alpha_{2}\right)\left(1-\mu_{1} \mu_{2} k_{1} k_{2}\right)} \int_{0}^{1} a(t) d A_{1}(t)+\frac{1}{\Gamma\left(\alpha_{2}\right)}, \frac{\mu_{1}}{\Gamma\left(\alpha_{2}\right)\left(1-\mu_{1} \mu_{2} k_{1} k_{2}\right)} \int_{0}^{1} a(t) d A_{1}(t),
\end{array}\right\} \\
& \varrho=\max \left\{\begin{array}{l}
\frac{\mu_{1} \mu_{2} k_{1}}{\Gamma\left(\alpha_{1}\right)\left(1-\mu_{1} \mu_{2} k_{1} k_{2}\right)} \int_{0}^{1} b(t)\left(1-t^{\alpha_{1}-1}\right) d A_{2}(t), \\
\frac{\mu_{2}}{\Gamma\left(\alpha_{1}\right)\left(1-\mu_{1} \mu_{2} k_{1} k_{2}\right)} \int_{0}^{1} b(t)\left(1-t^{\alpha_{1}-1}\right) d A_{2}(t), \\
\frac{\mu_{1} \mu_{2} k_{2}}{\Gamma\left(\alpha_{2}\right)\left(1-\mu_{1} \mu_{2} k_{1} k_{2}\right)} \int_{0}^{1} a(t)\left(1-t^{\alpha_{2}-1}\right) d A_{1}(t), \\
\frac{\mu_{1}}{\Gamma\left(\alpha_{2}\right)\left(1-\mu_{1} \mu_{2} k_{1} k_{2}\right)} \int_{0}^{1} a(t)\left(1-t^{\alpha_{2}-1}\right) d A_{1}(t) .
\end{array}\right\}
\end{aligned}
$$

Remark 2.1 From Lemma 2.4, for $t, \tau, s \in[0,1]$, we have

$$
\begin{array}{ll}
K_{i}(t, s) \geq \omega K_{i}(\tau, s), & H_{i}(t, s) \geq \omega H_{i}(\tau, s), \quad i=1,2, \\
K_{1}(t, s) \geq \omega H_{2}(\tau, s), & H_{2}(t, s) \geq \omega K_{1}(\tau, s), \\
K_{2}(t, s) \geq \omega H_{1}(\tau, s), & H_{1}(t, s) \geq \omega K_{2}(\tau, s),
\end{array}
$$

where $\omega=\frac{\varrho}{\rho}, \varrho, \rho$ are defined as Lemma $2.4,0<\omega<1$.

Let $X=C[0,1] \times C[0,1]$, then $X$ is a Banach space with the norm

$$
\|(u, v)\|=\max \{\|u\|,\|v\|\}, \quad\|u\|=\max _{t \in[0,1]}|u(t)|, \quad\|v\|=\max _{t \in[0,1]}|v(t)| .
$$

Let

$$
K=\{(u, v) \in X: u(t) \geq \omega\|(u, v)\|, v(t) \geq \omega\|(u, v)\|, t \in[0,1]\},
$$

where $\omega$ is defined as Remark 2.1. It is easy to see that $K$ is a positive cone in $X$. For any $(u, v) \in K$, we can define an integral operator $T: K \rightarrow X$ by

$$
\begin{aligned}
T(u, v)(t)= & \left(T_{1}(u, v)(t), T_{2}(u, v)(t)\right), \quad 0 \leq t \leq 1, \\
T_{1}(u, v)(t)= & \int_{0}^{1} K_{1}(t, s) \varphi_{q_{1}}\left(\int_{0}^{1} \bar{H}_{1}(s, \tau) f_{1}(\tau, u(\tau), v(\tau)) d \tau\right) d s \\
& +\int_{0}^{1} H_{1}(t, s) \varphi_{q_{2}}\left(\int_{0}^{1} \bar{H}_{2}(s, \tau) f_{2}(\tau, u(\tau), v(\tau)) d \tau\right) d s, \quad 0 \leq t \leq 1, \\
T_{2}(u, v)(t)= & \int_{0}^{1} K_{2}(t, s) \varphi_{q_{2}}\left(\int_{0}^{1} \bar{H}_{2}(s, \tau) f_{2}(\tau, u(\tau), v(\tau)) d \tau\right) d s \\
& +\int_{0}^{1} H_{2}(t, s) \varphi_{q_{1}}\left(\int_{0}^{1} \bar{H}_{1}(s, \tau) f_{1}(\tau, u(\tau), v(\tau)) d \tau\right) d s, \quad 0 \leq t \leq 1 .
\end{aligned}
$$

We know that $(u, v)$ is a positive solutions of system $(1.1)$ if and only if $(u, v)$ is a fixed point of $T$ in $K$.

Lemma 2.5 $T: X \rightarrow X$ is a completely continuous operator and $T(K) \subseteq K$. 
Proof By a routine discussion, we see that $T: X \rightarrow X$ is well defined, so we only prove $T(K) \subseteq K$. For any $(u, v) \in K, 0 \leq t, t^{\prime} \leq 1$, by Remark 2.1, we have

$$
\begin{aligned}
T_{1}(u, v)(t)= & \int_{0}^{1} K_{1}(t, s) \varphi_{q_{1}}\left(\int_{0}^{1} \bar{H}_{1}(s, \tau) f_{1}(\tau, u(\tau), v(\tau)) d \tau\right) d s \\
& +\int_{0}^{1} H_{1}(t, s) \varphi_{q_{2}}\left(\int_{0}^{1} \bar{H}_{2}(s, \tau) f_{2}(\tau, u(\tau), v(\tau)) d \tau\right) d s \\
\geq & \int_{0}^{1} \omega K_{1}\left(t^{\prime}, s\right) \varphi_{q_{1}}\left(\int_{0}^{1} \bar{H}_{1}(s, \tau) f_{1}(\tau, u(\tau), v(\tau)) d \tau\right) d s \\
& +\int_{0}^{1} \omega H_{1}\left(t^{\prime}, s\right) \varphi_{q_{2}}\left(\int_{0}^{1} \bar{H}_{2}(s, \tau) f_{2}(\tau, u(\tau), v(\tau)) d \tau\right) d s \\
\geq & \omega\left(\int_{0}^{1} K_{1}\left(t^{\prime}, s\right) \varphi_{q_{1}}\left(\int_{0}^{1} \bar{H}_{1}(s, \tau) f_{1}(\tau, u(\tau), v(\tau)) d \tau\right) d s\right. \\
& \left.+\int_{0}^{1} H_{1}\left(t^{\prime}, s\right) \varphi_{q_{2}}\left(\int_{0}^{1} \bar{H}_{2}(s, \tau) f_{2}(\tau, u(\tau), v(\tau)) d \tau\right) d s\right) \\
\geq & \omega T_{1}(u, v)\left(t^{\prime}\right), \\
T_{1}(u, v)(t) \geq & \int_{0}^{1} \omega H_{2}\left(t^{\prime}, s\right) \varphi_{q_{1}}\left(\int_{0}^{1} \bar{H}_{1}(s, \tau) f_{1}(\tau, u(\tau), v(\tau)) d \tau\right) d s \\
& +\int_{0}^{1} \omega K_{2}\left(t^{\prime}, s\right)\left(\int_{0}^{1} \bar{H}_{2}(s, \tau) f_{2}(\tau, u(\tau), v(\tau)) d \tau\right) d s \\
\geq & \omega\left(\int_{0}^{1} H_{2}\left(t^{\prime}, s\right) \varphi_{q_{1}}\left(\int_{0}^{1} \bar{H}_{1}(s, \tau) f_{1}(\tau, u(\tau), v(\tau)) d \tau\right) d s\right. \\
& \left.+\int_{0}^{1} K_{2}\left(t^{\prime}, s\right)\left(\int_{0}^{1} \bar{H}_{2}(s, \tau) f_{2}(\tau, u(\tau), v(\tau)) d \tau\right)\right) \\
\geq & \omega T_{2}(u, v)\left(t^{\prime}\right) .
\end{aligned}
$$

So we have

$$
T_{1}(u, v)(t) \geq \omega\left\|T_{1}(u, v)\right\|, \quad T_{1}(u, v)(t) \geq \omega\left\|T_{2}(u, v)\right\|,
$$

i.e.,

$$
T_{1}(u, v)(t) \geq \omega\left\|\left(T_{1}(u, v), T_{2}(u, v)\right)\right\| .
$$

In the same way as (2.11) and (2.12), we can prove that

$$
T_{2}(u, v)(t) \geq \omega\left\|\left(T_{1}(u, v), T_{2}(u, v)\right)\right\| .
$$

Therefore, we have $T(K) \subseteq K$.

According to the Ascoli-Arzela theorem, we see that $T: K \rightarrow K$ is completely continuous. The proof is completed.

Lemma 2.6 ([45]) Let $K$ be a positive cone in a Banach space $E, \Omega_{1}$ and $\Omega_{2}$ are bounded open sets in $E, \theta \in \Omega_{1}, \bar{\Omega}_{1} \subset \Omega_{2}, T: K \cap \bar{\Omega}_{2} \backslash \Omega_{1} \rightarrow K$ is a completely continuous operator. 
If the following conditions are satisfied:

$$
\|T x\| \leq\|x\|, \quad \forall x \in K \cap \partial \Omega_{1}, \quad\|T x\| \geq\|x\|, \quad \forall x \in K \cap \partial \Omega_{2},
$$

or

$$
\|T x\| \geq\|x\|, \quad \forall x \in K \cap \partial \Omega_{1}, \quad\|T x\| \leq\|x\|, \quad \forall x \in K \cap \partial \Omega_{2},
$$

then $T$ has at least one fixed point in $K \cap\left(\bar{\Omega}_{2} \backslash \Omega_{1}\right)$.

\section{Main results}

Denote

$$
\begin{aligned}
& f_{10}=\liminf _{x \rightarrow 0^{+}} \inf _{\substack{t \in[a, b] \subset(0,1) \\
y \in[0,+\infty)}} \frac{f_{1}(t, x, y)}{\varphi_{p_{1}}(x)}, \quad f_{1}^{0}=\limsup _{x \rightarrow 0^{+}} \sup _{\substack{t \in[0,1] \\
y \in[0,+\infty)}} \frac{f_{1}(t, x, y)}{\varphi_{p_{1}}(x)}, \\
& f_{20}=\liminf _{y \rightarrow 0^{+}} \inf _{\substack{t \in[a, b] \subset(0,1) \\
x \in[0,+\infty)}} \frac{f_{2}(t, x, y)}{\varphi_{p_{2}}(y)}, \quad f_{2}^{0}=\limsup _{y \rightarrow 0^{+}} \sup _{\substack{t \in[0,1] \\
x \in[0,+\infty)}} \frac{f_{2}(t, x, y)}{\varphi_{p_{2}}(y)}, \\
& f_{1 \infty}=\liminf _{x \rightarrow+\infty} \inf _{\substack{t \in[a, b] c(0,1) \\
y \in[0,+\infty)}} \frac{f_{1}(t, x, y)}{\varphi_{p_{1}}(x)}, \quad f_{1}^{\infty}=\limsup _{x \rightarrow+\infty} \sup _{\substack{t \in[0,1] \\
y \in[0,+\infty)}} \frac{f_{1}(t, x, y)}{\varphi_{p_{1}}(x)}, \\
& f_{2 \infty}=\liminf _{y \rightarrow+\infty} \inf _{\substack{t \in[a, b] \subset(0,1) \\
x \in[0,+\infty)}} \frac{f_{2}(t, x, y)}{\varphi_{p_{2}}(y)}, \quad f_{2}^{\infty}=\limsup _{y \rightarrow+\infty} \sup _{\substack{t \in[0,1] \\
x \in[0,+\infty)}} \frac{f_{2}(t, x, y)}{\varphi_{p_{2}}(y)}, \\
& L_{i}=\left(\frac{1}{2} \int_{0}^{1} \rho(1-s)^{\alpha_{i}-1} \varphi_{q_{i}}\left(\int_{0}^{1} \bar{H}_{i}(s, \tau) d \tau\right) d s\right)^{-1}, \\
& l_{i}=\left(\frac{1}{2} \int_{0}^{1} \varrho(1-s)^{\alpha_{i}-1} \varphi_{q_{i}}\left(\int_{a}^{b} \bar{H}_{i}(s, \tau) d \tau\right) d s\right)^{-1}, \quad i=1,2 .
\end{aligned}
$$

In what follows, we list the conditions to be used later:

$\left(\mathbf{H}_{1}\right) f_{i 0} \in\left(\varphi_{p_{i}}\left(\frac{l_{i}}{\omega}\right),+\infty\right], f_{i \infty} \in\left(\varphi_{p_{i}}\left(\frac{l_{i}}{\omega}\right),+\infty\right]$.

$\left(\mathbf{H}_{2}\right) f_{i}^{0} \in\left[0, \varphi_{p_{i}}\left(L_{i}\right)\right), f_{i}^{\infty} \in\left[0, \varphi_{p_{i}}\left(L_{i}\right)\right)$.

$\left(\mathbf{H}_{3}\right)$ There exist constants $d_{i} \in\left(0, L_{i}\right)$ and $r_{1}>0$, such that

$$
f_{i}(t, x, y) \leq \varphi_{p_{i}}\left(d_{i} r_{1}\right), \quad 0 \leq t \leq 1,0 \leq x, y \leq r_{1} .
$$

$\left(\mathbf{H}_{4}\right)$ There exist constants $d_{i}^{*} \in\left(l_{i},+\infty\right)$ and $R_{1}>0,[a, b] \subset(0,1)$, such that

$$
f_{i}(t, x, y) \geq \varphi_{p_{i}}\left(d_{i}^{*} R_{1}\right), \quad a \leq t \leq b, \omega R_{1} \leq x, y \leq R_{1} .
$$

Theorem 3.1 Assume that $\left(\mathbf{H}_{0}\right),\left(\mathbf{H}_{1}\right),\left(\mathbf{H}_{3}\right)$ hold, then system (1.1) has at least two positive solutions $\left(u_{1}, v_{1}\right)$ and $\left(u_{2}, v_{2}\right)$ such that $0<\left\|\left(u_{1}, v_{1}\right)\right\|<r_{1}<\left\|\left(u_{2}, v_{2}\right)\right\|$.

Proof (I) By $\left(\mathbf{H}_{3}\right)$, there exist constants $d_{i} \in\left(0, L_{i}\right)$ and $r_{1}>0$, such that

$$
f_{i}(t, x, y) \leq \varphi_{p_{i}}\left(d_{i} r_{1}\right), \quad 0 \leq t \leq 1,0 \leq x, y \leq r_{1} .
$$


Let $K_{r_{1}}=\left\{(u, v) \in K:\|(u, v)\|<r_{1}\right\}$. For any $(u, v) \in \partial K_{r_{1}}$, by the definition of $\|\cdot\|$, we know that

$$
\begin{aligned}
& u(t) \leq|u(t)| \leq\|u\| \leq\|(u, v)\| \leq r_{1}, \\
& v(t) \leq|v(t)| \leq\|v\| \leq\|(u, v)\| \leq r_{1}, \quad 0 \leq t \leq 1 .
\end{aligned}
$$

Thus, for any $(u, v) \in \partial K_{r_{1}}$, by (3.1) and (3.2), we can obtain

$$
f_{i}(t, u(t), v(t)) \leq \varphi_{p_{i}}\left(d_{i} r_{1}\right), \quad 0 \leq t \leq 1
$$

Hence, for any $(u, v) \in \partial K_{r_{1}}$, by Lemmas 2.3, 2.4 and (3.3), we have

$$
\begin{aligned}
T_{1}(u, v)(t)= & \int_{0}^{1} K_{1}(t, s) \varphi_{q_{1}}\left(\int_{0}^{1} \bar{H}_{1}(s, \tau) f_{1}(\tau, u(\tau), v(\tau)) d \tau\right) d s \\
& +\int_{0}^{1} H_{1}(t, s) \varphi_{q_{2}}\left(\int_{0}^{1} \bar{H}_{2}(s, \tau) f_{2}(\tau, u(\tau), v(\tau)) d \tau\right) d s \\
\leq & \int_{0}^{1} \rho(1-s)^{\alpha_{1}-1} \varphi_{q_{1}}\left(\int_{0}^{1} \bar{H}_{1}(s, \tau) f_{1}(\tau, u(\tau), v(\tau)) d \tau\right) d s \\
& +\int_{0}^{1} \rho(1-s)^{\alpha_{2}-1} \varphi_{q_{2}}\left(\int_{0}^{1} \bar{H}_{2}(s, \tau) f_{2}(\tau, u(\tau), v(\tau)) d \tau\right) d s \\
\leq & \int_{0}^{1} \rho(1-s)^{\alpha_{1}-1} \varphi_{q_{1}}\left(\int_{0}^{1} \bar{H}_{1}(s, \tau) \varphi_{p_{1}}\left(d_{1} r_{1}\right) d \tau\right) d s \\
& +\int_{0}^{1} \rho(1-s)^{\alpha_{2}-1} \varphi_{q_{2}}\left(\int_{0}^{1} \bar{H}_{2}(s, \tau) \varphi_{p_{2}}\left(d_{2} r_{1}\right) d \tau\right) d s \\
\leq & r_{1}\left(L_{1} \int_{0}^{1} \rho(1-s)^{\alpha_{1}-1} \varphi_{q_{1}}\left(\int_{0}^{1} \bar{H}_{1}(s, \tau) d \tau\right) d s\right. \\
& \left.+L_{2} \int_{0}^{1} \rho(1-s)^{\alpha_{2}-1} \varphi_{q_{2}}\left(\int_{0}^{1} \bar{H}_{2}(s, \tau) d \tau\right) d s\right) \\
= & r_{1}=\|(u, v)\| .
\end{aligned}
$$

Similar to (3.4), for any $(u, v) \in \partial K_{r_{1}}$, we also have

$$
\left\|T_{2}(u, v)\right\| \leq r_{1}=\|(u, v)\| .
$$

\section{Consequently}

$$
\|T(u, v)\|=\max \left\{\left\|T_{1}(u, v)\right\|,\left\|T_{2}(u, v)\right\|\right\} \leq r_{1}=\|(u, v)\|, \quad(u, v) \in \partial K_{r_{1}} .
$$

(II) With the first inequality of $\left(\mathbf{H}_{1}\right), f_{i 0} \in\left(\varphi_{p_{i}}\left(\frac{l_{i}}{\omega}\right),+\infty\right]$, there exists a real number $r \in$ $\left(0, r_{1}\right)$, such that

$$
\begin{aligned}
& f_{1}(t, x, y) \leq \varphi_{p_{1}}(x) \varphi_{p_{1}}\left(\frac{l_{1}}{\omega}\right), \quad a \leq t \leq b, 0 \leq x \leq r, y \geq 0, \\
& f_{2}(t, x, y) \leq \varphi_{p_{2}}(y) \varphi_{p_{2}}\left(\frac{l_{2}}{\omega}\right), \quad a \leq t \leq b, 0 \leq y \leq r, x \geq 0 .
\end{aligned}
$$


Let $K_{r}=\{(u, v) \in K:\|(u, v)\|<r\}$. For any $(u, v) \in \partial K_{r}$,

$$
\begin{aligned}
& r=\|(u, v)\| \geq u(t) \geq \omega\|(u, v)\| \geq \omega r, \\
& r=\|(u, v)\| \geq v(t) \geq \omega\|(u, v)\| \geq \omega r, \quad 0 \leq t \leq 1 .
\end{aligned}
$$

By Lemmas 2.3, 2.4 and (3.6), (3.7), we have

$$
\begin{aligned}
T_{1}(u, v)(t)= & \int_{0}^{1} K_{1}(t, s) \varphi_{q_{1}}\left(\int_{0}^{1} \bar{H}_{1}(s, \tau) f_{1}(\tau, u(\tau), v(\tau)) d \tau\right) d s \\
& +\int_{0}^{1} H_{1}(t, s) \varphi_{q_{2}}\left(\int_{0}^{1} \bar{H}_{2}(s, \tau) f_{2}(\tau, u(\tau), v(\tau)) d \tau\right) d s \\
\geq & \int_{0}^{1} \varrho(1-s)^{\alpha_{1}-1} \varphi_{q_{1}}\left(\int_{0}^{1} \bar{H}_{1}(s, \tau) f_{1}(\tau, u(\tau), v(\tau)) d \tau\right) d s \\
& +\int_{0}^{1} \varrho(1-s)^{\alpha_{2}-1} \varphi_{q_{2}}\left(\int_{0}^{1} \bar{H}_{2}(s, \tau) f_{2}(\tau, u(\tau), v(\tau)) d \tau\right) d s \\
\geq & \int_{0}^{1} \varrho(1-s)^{\alpha_{1}-1} \varphi_{q_{1}}\left(\int_{a}^{b} \bar{H}_{1}(s, \tau) \varphi_{p_{1}}(u(\tau)) \varphi_{p_{1}}\left(\frac{l_{1}}{\omega}\right) d \tau\right) d s \\
& +\int_{0}^{1} \varrho(1-s)^{\alpha_{2}-1} \varphi_{q_{2}}\left(\int_{a}^{b} \bar{H}_{2}(s, \tau) \varphi_{p_{2}}(v(\tau)) \varphi_{p_{2}}\left(\frac{l_{2}}{\omega}\right) d \tau\right) d s \\
\geq & r\left(l_{1} \int_{0}^{1} \varrho(1-s)^{\alpha_{1}-1} \varphi_{q_{1}}\left(\int_{a}^{b} \bar{H}_{1}(s, \tau) d \tau\right) d s\right. \\
& \left.+l_{2} \int_{0}^{1} \varrho(1-s)^{\alpha_{2}-1} \varphi_{q_{2}}\left(\int_{a}^{b} \bar{H}_{2}(s, \tau) d \tau\right) d s\right) \\
= & r=\|(u, v)\| .
\end{aligned}
$$

Therefore, we obtain

$$
\|T(u, v)\|=\max \left\{\left\|T_{1}(u, v)\right\|,\left\|T_{2}(u, v)\right\|\right\} \geq r=\|(u, v)\|, \quad \text { for any }(u, v) \in \partial K_{r} .
$$

(III) With the second inequality of $\left(\mathbf{H}_{1}\right), f_{i \infty} \in\left(\varphi_{p_{i}}\left(\frac{l_{i}}{\omega}\right),+\infty\right]$, there exist real numbers $r_{2}^{*}$, $r_{2}^{* *}$, such that

$$
\begin{array}{ll}
f_{1}(t, x, y) \geq \varphi_{p_{1}}(x) \varphi_{p_{1}}\left(\frac{l_{1}}{\omega}\right), & a \leq t \leq b, x \geq r_{2}^{*}, y \geq 0, \\
f_{2}(t, x, y) \geq \varphi_{p_{2}}(y) \varphi_{p_{2}}\left(\frac{l_{2}}{\omega}\right), & a \leq t \leq b, y \geq r_{2}^{* *}, x \geq 0 .
\end{array}
$$

Choose $r_{2}=\max \left\{2 r_{1}, \frac{r^{*}}{\omega \theta}, \frac{r_{2}^{* *}}{\omega \theta}\right\}$. Let $K_{r_{2}}=\left\{(u, v) \in K:\|(u, v)\|<r_{2}\right\}$. For any $(u, v) \in \partial K_{r_{2}}$, by the definition of $\|\cdot\|$, we have

$$
\begin{aligned}
& r_{2}=\|(u, v)\| \geq u(t) \geq \omega\|(u, v)\| \geq \omega r_{2} \geq r_{2}^{*}, \quad 0 \leq t \leq 1, \\
& r_{2}=\|(u, v)\| \geq v(t) \geq \omega\|(u, v)\| \geq \omega r_{2} \geq r_{2}^{* *}, \quad 0 \leq t \leq 1 .
\end{aligned}
$$


Thus, for any $(u, v) \in \partial K_{r_{2}}$, by (3.10), (3.11), we have

$$
\begin{array}{ll}
f_{1}(t, u(t), v(t)) \geq \varphi_{p_{1}}(u(t)) \varphi_{p_{1}}\left(\frac{l_{1}}{\omega}\right) \geq \varphi_{p_{1}}\left(\omega r_{2}\right) \varphi_{p_{1}}\left(\frac{l_{1}}{\omega}\right), & a \leq t \leq b, \\
f_{2}(t, u(t), v(t)) \geq \varphi_{p_{2}}(v(t)) \varphi_{p_{2}}\left(\frac{l_{2}}{\omega}\right) \geq \varphi_{p_{2}}\left(\omega r_{2}\right) \varphi_{p_{2}}\left(\frac{l_{2}}{\omega}\right), \quad a \leq t \leq b .
\end{array}
$$

So, for any $(u, v) \in \partial K_{r_{2}}$, by Lemmas $2.3,2.4$ and (3.12), we know

$$
\begin{aligned}
T_{1}(u, v)(t) \geq & \int_{0}^{1} \varrho(1-s)^{\alpha_{1}-1} \varphi_{q_{1}}\left(\int_{0}^{1} \bar{H}_{1}(s, \tau) f_{1}(\tau, u(\tau), v(\tau)) d \tau\right) d s \\
& +\int_{0}^{1} \varrho(1-s)^{\alpha_{2}-1} \varphi_{q_{2}}\left(\int_{0}^{1} \bar{H}_{2}(s, \tau) f_{2}(\tau, u(\tau), v(\tau)) d \tau\right) d s \\
\geq & \int_{0}^{1} \varrho(1-s)^{\alpha_{1}-1} \varphi_{q_{1}}\left(\int_{a}^{b} \bar{H}_{1}(s, \tau) \varphi_{p_{1}}(u(\tau)) \varphi_{p_{1}}\left(\frac{l_{1}}{\omega}\right) d \tau\right) d s \\
& +\int_{0}^{1} \varrho(1-s)^{\alpha_{2}-1} \varphi_{q_{2}}\left(\int_{a}^{b} \bar{H}_{2}(s, \tau) \varphi_{p_{2}}(v(\tau)) \varphi_{p_{2}}\left(\frac{l_{2}}{\omega}\right) d \tau\right) d s \\
\geq & \int_{0}^{1} \varrho(1-s)^{\alpha_{1}-1} \varphi_{q_{1}}\left(\int_{a}^{b} \bar{H}_{1}(s, \tau) \varphi_{p_{1}}\left(\omega r_{2}\right) \varphi_{p_{1}}\left(\frac{l_{1}}{\omega}\right) d \tau\right) d s \\
& +\int_{0}^{1} \varrho(1-s)^{\alpha_{2}-1} \varphi_{q_{2}}\left(\int_{a}^{b} \bar{H}_{2}(s, \tau) \varphi_{p_{2}}\left(\omega r_{2}\right) \varphi_{p_{2}}\left(\frac{l_{2}}{\omega}\right) d \tau\right) d s \\
\geq & r_{2}\left(l_{1} \int_{0}^{1} \varrho(1-s)^{\alpha_{1}-1} \varphi_{q_{1}}\left(\int_{a}^{b} \bar{H}_{1}(s, \tau) d \tau\right) d s\right. \\
& \left.+l_{2} \int_{0}^{1} \varrho(1-s)^{\alpha_{2}-1} \varphi_{q_{2}}\left(\int_{a}^{b} \bar{H}_{2}(s, \tau) d \tau\right) d s\right) \\
= & r_{2}=\|(u, v)\| .
\end{aligned}
$$

Hence, we obtain

$$
\|T(u, v)\|=\max \left\{\left\|T_{1}(u, v)\right\|,\left\|T_{2}(u, v)\right\|\right\} \geq r_{2}=\|(u, v)\|, \quad \text { for any }(u, v) \in \partial K_{r_{2}} .
$$

It follows from the above discussion, (3.5), (3.9), (3.14), Lemmas 2.5, 2.6, that $T$ has fixed points $\left(u_{1}, v_{1}\right) \in \bar{K}_{r_{2}} \backslash K_{r},\left(u_{2}, v_{2}\right) \in \bar{K}_{r} \backslash K_{r_{1}}$, that is to say, system (1.1) has at least two positive solutions $\left(u_{1}, v_{1}\right),\left(u_{2}, v_{2}\right)$, satisfying $0<\left\|\left(u_{1}, v_{1}\right)\right\|<r_{1}<\left\|\left(u_{2}, v_{2}\right)\right\|$. The proof is completed.

Theorem 3.2 Assume that $\left(\mathbf{H}_{0}\right),\left(\mathbf{H}_{2}\right),\left(\mathbf{H}_{4}\right)$ hold, then system (1.1) has at least two positive solutions $\left(u_{1}, v_{1}\right)$ and $\left(u_{2}, v_{2}\right)$ such that $0<\left\|\left(u_{1}, v_{1}\right)\right\|<R_{1}<\left\|\left(u_{2}, v_{2}\right)\right\|$.

Proof (I) By $\left(\mathbf{H}_{4}\right)$, there exist constants $d_{i}^{*} \in\left(l_{i},+\infty\right)$ and $R_{1}>0$, such that

$$
f_{i}(t, x, y) \geq \varphi_{p_{i}}\left(d_{i}^{*} R_{1}\right), \quad a \leq t \leq b, \omega R_{0} \leq x, y \leq R_{1} .
$$


Let $K_{R_{1}}=\left\{(u, v) \in K:\|(u, v)\|<R_{1}\right\}$. For any $(u, v) \in \partial K_{R_{1}}$,

$$
\begin{aligned}
& R_{1}=\|(u, v)\| \geq u(t) \geq \omega\|(u, v)\| \geq \omega R_{1}, \\
& R_{1}=\|(u, v)\| \geq v(t) \geq \omega\|(u, v)\| \geq \omega R_{1}, \quad 0 \leq t \leq 1 .
\end{aligned}
$$

Thus, for any $(u, v) \in \partial K_{R_{1}}$, by Lemmas 2.3, 2.4 and (3.15), (3.16), we get

$$
\begin{aligned}
T_{1}(u, v)(t)= & \int_{0}^{1} K_{1}(t, s) \varphi_{q_{1}}\left(\int_{0}^{1} \bar{H}_{1}(s, \tau) f_{1}(\tau, u(\tau), v(\tau)) d \tau\right) d s \\
& +\int_{0}^{1} H_{1}(t, s) \varphi_{q_{2}}\left(\int_{0}^{1} \bar{H}_{2}(s, \tau) f_{2}(\tau, u(\tau), v(\tau)) d \tau\right) d s \\
\geq & \int_{0}^{1} \varrho(1-s)^{\alpha_{1}-1} \varphi_{q_{1}}\left(\int_{0}^{1} \bar{H}_{1}(s, \tau) f_{1}(\tau, u(\tau), v(\tau)) d \tau\right) d s \\
& +\int_{0}^{1} \varrho(1-s)^{\alpha_{2}-1} \varphi_{q_{2}}\left(\int_{0}^{1} \bar{H}_{2}(s, \tau) f_{2}(\tau, u(\tau), v(\tau)) d \tau\right) d s \\
\geq & \int_{0}^{1} \varrho(1-s)^{\alpha_{1}-1} \varphi_{q_{1}}\left(\int_{a}^{b} \bar{H}_{1}(s, \tau) \varphi_{p_{1}}\left(d_{1}^{*} R_{0}\right) d \tau\right) d s \\
& +\int_{0}^{1} \varrho(1-s)^{\alpha_{2}-1} \varphi_{q_{2}}\left(\int_{a}^{b} \bar{H}_{2}(s, \tau) \varphi_{p_{2}}\left(d_{2}^{*} R_{0}\right) d \tau\right) d s \\
\geq & R_{1}\left(l_{1} \int_{0}^{1} \varrho(1-s)^{\alpha_{1}-1} \varphi_{q_{1}}\left(\int_{a}^{b} \bar{H}_{1}(s, \tau) d \tau\right) d s\right. \\
& \left.+l_{2} \int_{0}^{1} \varrho(1-s)^{\alpha_{2}-1} \varphi_{q_{2}}\left(\int_{a}^{b} \bar{H}_{2}(s, \tau) d \tau\right) d s\right) \\
= & R_{1}=\|(u, v)\| .
\end{aligned}
$$

So, we have

$$
\begin{aligned}
\|T(u, v)\| & =\max \left\{\left\|T_{1}(u, v)\right\|,\left\|T_{2}(u, v)\right\|\right\} \\
& \geq R_{1}=\|(u, v)\|, \quad \text { for any }(u, v) \in \partial K_{R_{1}} .
\end{aligned}
$$

(II) With the first inequality of $\left(\mathbf{H}_{2}\right), f_{i}^{0} \in\left[0, \varphi_{p_{i}}\left(L_{i}\right)\right)$, there exists a real number $R_{2} \in$ $\left(0, R_{1}\right)$, such that

$$
\begin{array}{ll}
f_{1}(t, x, y) \leq \varphi_{p_{1}}\left(x L_{1}\right) \leq \varphi_{p_{1}}\left(R_{2} L_{1}\right), & 0 \leq t \leq 1,0 \leq x \leq R_{2}, y \geq 0, \\
f_{2}(t, x, y) \leq \varphi_{p_{2}}\left(y L_{2}\right) \leq \varphi_{p_{2}}\left(R_{2} L_{2}\right), & 0 \leq t \leq 1,0 \leq y \leq R_{2}, x \geq 0 .
\end{array}
$$

Let $K_{R_{2}}=\left\{(u, v) \in K:\|(u, v)\|<R_{2}\right\}$. For any $(u, v) \in \partial K_{R_{2}}$,

$$
\begin{aligned}
& u(t) \leq|u(t)| \leq\|u\| \leq\|(u, v)\| \leq R_{2}, \\
& v(t) \leq|v(t)| \leq\|v\| \leq\|(u, v)\| \leq R_{2}, \quad 0 \leq t \leq 1 .
\end{aligned}
$$


Therefore, for any $(u, v) \in \partial K_{R_{2}}$, by Lemmas 2.3, 2.4 and (3.19), (3.20), we have

$$
\begin{aligned}
T_{1}(u, v)(t)= & \int_{0}^{1} K_{1}(t, s) \varphi_{q_{1}}\left(\int_{0}^{1} \bar{H}_{1}(s, \tau) f_{1}(\tau, u(\tau), v(\tau)) d \tau\right) d s \\
& +\int_{0}^{1} H_{1}(t, s) \varphi_{q_{2}}\left(\int_{0}^{1} \bar{H}_{2}(s, \tau) f_{2}(\tau, u(\tau), v(\tau)) d \tau\right) d s \\
\leq & \int_{0}^{1} \rho(1-s)^{\alpha_{1}-1} \varphi_{q_{1}}\left(\int_{0}^{1} \bar{H}_{1}(s, \tau) f_{1}(\tau, u(\tau), v(\tau)) d \tau\right) d s \\
& +\int_{0}^{1} \rho(1-s)^{\alpha_{2}-1} \varphi_{q_{2}}\left(\int_{0}^{1} \bar{H}_{2}(s, \tau) f_{2}(\tau, u(\tau), v(\tau)) d \tau\right) d s \\
\leq & \int_{0}^{1} \rho(1-s)^{\alpha_{1}-1} \varphi_{q_{1}}\left(\int_{0}^{1} \bar{H}_{1}(s, \tau) \varphi_{p_{1}}\left(R_{2} L_{1}\right) d \tau\right) d s \\
& +\int_{0}^{1} \rho(1-s)^{\alpha_{2}-1} \varphi_{q_{2}}\left(\int_{0}^{1} \bar{H}_{2}(s, \tau) \varphi_{p_{2}}\left(R_{2} L_{2}\right) d \tau\right) d s \\
\leq & R_{2}\left(L_{1} \int_{0}^{1} \rho(1-s)^{\alpha_{1}-1} \varphi_{q_{1}}\left(\int_{0}^{1} \bar{H}_{1}(s, \tau) d \tau\right) d s\right. \\
& \left.+L_{2} \int_{0}^{1} \rho(1-s)^{\alpha_{2}-1} \varphi_{q_{2}}\left(\int_{0}^{1} \bar{H}_{2}(s, \tau) d \tau\right) d s\right) \\
= & R_{2}=\|(u, v)\| .
\end{aligned}
$$

By a similar proof to (3.21), for any $(u, v) \in \partial K_{R_{2}}$, we also have

$$
\left\|T_{2}(u, v)\right\| \leq R_{2}=\|(u, v)\|
$$

Thus,

$$
\|T(u, v)\|=\max \left\{\left\|T_{1}(u, v)\right\|,\left\|T_{2}(u, v)\right\|\right\} \leq R_{2}=\|(u, v)\|, \quad(u, v) \in \partial K_{R_{2}} .
$$

(III) With the second inequality of $\left(\mathbf{H}_{2}\right), f_{i}^{\infty} \in\left[0, \varphi_{p_{i}}\left(L_{i}\right)\right)$, there exists $R^{*}>0$, such that

$$
\begin{array}{ll}
f_{1}(t, x, y) \leq \varphi_{p_{1}}\left(x L_{1}\right), & 0 \leq t \leq 1, x \geq R^{*}, y \geq 0, \\
f_{2}(t, x, y) \leq \varphi_{p_{2}}\left(y L_{2}\right), & 0 \leq t \leq 1, y \geq R^{*}, x \geq 0 .
\end{array}
$$

Now there are two situations.

Case $1 . f_{i}$ is bounded on $[0,+\infty)$, then we choose $\bar{R}>0$, such that

$$
f_{i}(t, x, y) \leq \varphi_{p_{i}}\left(\bar{R} L_{i}\right), \quad 0 \leq t \leq 1, x, y \geq 0, i=1,2
$$

Let $R_{3}=\max \left\{2 R_{1}, \bar{R}\right\}, K_{R_{3}}=\left\{(u, v) \in K:\|(u, v)\|<R_{3}\right\}$. For any $(u, v) \in \partial K_{R_{3}}$, we know

$$
\begin{aligned}
T_{1}(u, v)(t) \leq & \int_{0}^{1} \rho(1-s)^{\alpha_{1}-1} \varphi_{q_{1}}\left(\int_{0}^{1} \bar{H}_{1}(s, \tau) f_{1}(\tau, u(\tau), v(\tau)) d \tau\right) d s \\
& +\int_{0}^{1} \rho(1-s)^{\alpha_{2}-1} \varphi_{q_{2}}\left(\int_{0}^{1} \bar{H}_{2}(s, \tau) f_{2}(\tau, u(\tau), v(\tau)) d \tau\right) d s
\end{aligned}
$$




$$
\begin{aligned}
\leq & \int_{0}^{1} \rho(1-s)^{\alpha_{1}-1} \varphi_{q_{1}}\left(\int_{0}^{1} \bar{H}_{1}(s, \tau) \varphi_{p_{1}}\left(R_{3} L_{1}\right) d \tau\right) d s \\
& +\int_{0}^{1} \rho(1-s)^{\alpha_{2}-1} \varphi_{q_{2}}\left(\int_{0}^{1} \bar{H}_{2}(s, \tau) \varphi_{p_{2}}\left(R_{3} L_{2}\right) d \tau\right) d s \\
\leq & R_{3}\left(L_{1} \int_{0}^{1} \rho(1-s)^{\alpha_{1}-1} \varphi_{q_{1}}\left(\int_{0}^{1} \bar{H}_{1}(s, \tau) d \tau\right) d s\right. \\
& \left.+L_{2} \int_{0}^{1} \rho(1-s)^{\alpha_{2}-1} \varphi_{q_{2}}\left(\int_{0}^{1} \bar{H}_{2}(s, \tau) d \tau\right) d s\right) \\
= & R_{3}=\|(u, v)\| .
\end{aligned}
$$

Similar to (3.25), for any $(u, v) \in \partial K_{R_{3}}$, we have

$$
\left\|T_{2}(u, v)\right\| \leq R_{3}=\|(u, v)\| .
$$

Thus,

$$
\|T(u, v)\|=\max \left\{\left\|T_{1}(u, v)\right\|,\left\|T_{2}(u, v)\right\|\right\} \leq R_{3}=\|(u, v)\|, \quad(u, v) \in \partial K_{R_{3}} .
$$

Case 2. $f_{1}$ and $f_{2}$ have at least one unbounded function, assume both $f_{1}$ and $f_{2}$ are unbounded. (If $f_{1}$ or $f_{2}$ is unbounded, the proof is similar.) Choose $R_{3}=\max \left\{2 R_{1}, \frac{R^{*}}{\omega}\right\}$, such that

$$
f_{i}(t, x, y) \leq f_{i}\left(t, R_{3}, R_{3}\right), \quad 0 \leq t \leq 1,0 \leq x, y \leq R_{3}, i=1,2 .
$$

Let $K_{R_{3}}=\left\{(u, v) \in K:\|(u, v)\|<R_{3}\right\}$. For any $(u, v) \in \partial K_{R_{3}}$, by (3.24), (3.27), we have

$$
\begin{aligned}
T_{1}(u, v)(t) \leq & \int_{0}^{1} \rho(1-s)^{\alpha_{1}-1} \varphi_{q_{1}}\left(\int_{0}^{1} \bar{H}_{1}(s, \tau) f_{1}(\tau, u(\tau), v(\tau)) d \tau\right) d s \\
& +\int_{0}^{1} \rho(1-s)^{\alpha_{2}-1} \varphi_{q_{2}}\left(\int_{0}^{1} \bar{H}_{2}(s, \tau) f_{2}(\tau, u(\tau), v(\tau)) d \tau\right) d s \\
\leq & \int_{0}^{1} \rho(1-s)^{\alpha_{1}-1} \varphi_{q_{1}}\left(\int_{0}^{1} \bar{H}_{1}(s, \tau) f_{1}\left(\tau, R_{3}, R_{3}\right) d \tau\right) d s \\
& +\int_{0}^{1} \rho(1-s)^{\alpha_{2}-1} \varphi_{q_{2}}\left(\int_{0}^{1} \bar{H}_{2}(s, \tau) f_{2}\left(\tau, R_{3}, R_{3}\right) d \tau\right) d s \\
\leq & \int_{0}^{1} \rho(1-s)^{\alpha_{1}-1} \varphi_{q_{1}}\left(\int_{0}^{1} \bar{H}_{1}(s, \tau) \varphi_{p_{1}}\left(R_{3} L_{1}\right) d \tau\right) d s \\
& +\int_{0}^{1} \rho(1-s)^{\alpha_{2}-1} \varphi_{q_{2}}\left(\int_{0}^{1} \bar{H}_{2}(s, \tau) \varphi_{p_{2}}\left(R_{3} L_{2}\right) d \tau\right) d s \\
\leq & R_{3}\left(L_{1} \int_{0}^{1} \rho(1-s)^{\alpha_{1}-1} \varphi_{q_{1}}\left(\int_{0}^{1} \bar{H}_{1}(s, \tau) d \tau\right) d s\right. \\
& \left.+L_{2} \int_{0}^{1} \rho(1-s)^{\alpha_{2}-1} \varphi_{q_{2}}\left(\int_{0}^{1} \bar{H}_{2}(s, \tau) d \tau\right) d s\right) \\
= & R_{3}=\|(u, v)\| .
\end{aligned}
$$


Similar to (3.28), for any $(u, v) \in \partial K_{R_{3}}$, we have

$$
\left\|T_{2}(u, v)\right\| \leq R_{3}=\|(u, v)\|
$$

Thus,

$$
\|T(u, v)\|=\max \left\{\left\|T_{1}(u, v)\right\|,\left\|T_{2}(u, v)\right\|\right\} \leq R_{3}=\|(u, v)\|, \quad(u, v) \in \partial K_{R_{3}} .
$$

Through the above discussion, (3.18), (3.22), (3.26) (or (3.29)), Lemmas 2.5, 2.6, $T$ has fixed points $\left(u_{1}, v_{1}\right) \in \bar{K}_{R_{1}} \backslash K_{R_{2}},\left(u_{2}, v_{2}\right) \in \bar{K}_{R_{3}} \backslash K_{R_{1}}$, that is to say, system (1.1) has at least two positive solutions $\left(u_{1}, v_{1}\right),\left(u_{2}, v_{2}\right)$, satisfying $0<\left\|\left(u_{1}, v_{1}\right)\right\|<R_{1}<\left\|\left(u_{2}, v_{2}\right)\right\|$. The proof is completed.

\section{An example}

Consider the following fractional differential system:

$$
\left\{\begin{array}{l}
D^{\frac{3}{2}}\left({ }^{c} D^{\frac{5}{2}} u(t)\right)+f_{1}(t, u(t), v(t))=0, \\
D^{\frac{3}{2}}\left({ }^{c} D^{\frac{5}{2}} v(t)\right)+f_{2}(t, u(t), v(t))=0, \quad 0<t<1, \\
u^{\prime}(0)=u^{\prime \prime}(0)=0, \quad v^{\prime}(0)=v^{\prime \prime}(0)=0, \\
u(1)=\frac{1}{2} \int_{0}^{1} s^{2} v(s) d s^{\frac{1}{3}}, \quad v(1)=\int_{0}^{1} s u(s) d s, \\
{ }^{c} D^{\frac{5}{2}} u(0)=0, \quad{ }^{c} D^{\frac{5}{2}} u(1)=\frac{1^{c}}{4} D^{\alpha_{1}} u\left(\frac{1}{2}\right), \\
{ }^{c} D^{\frac{5}{2}} v(0)=0, \quad{ }^{c} D^{\frac{5}{2}} v(1)=\frac{1^{c}}{4} D^{\alpha_{2}} v\left(\frac{1}{2}\right),
\end{array}\right.
$$

where $\beta_{1}=\beta_{2}=\frac{3}{2}, \alpha_{1}=\alpha_{2}=\frac{5}{2}, \mu_{1}=\frac{1}{2}, \mu_{2}=1, A_{1}(t)=t^{\frac{1}{3}}, A_{2}(t)=t, \varepsilon_{1}=\varepsilon_{2}=\frac{1}{4}, \eta_{1}=\eta_{2}=\frac{1}{2}$, $a(s)=s^{2}, b(s)=s, p_{1}=p_{2}=2$. Then we have

$$
\begin{aligned}
& k_{1}=\int_{0}^{1} a(s) d A_{1}(s)=\int_{0}^{1} s^{2} d s^{\frac{1}{3}}=\frac{1}{7}>0, \\
& k_{2}=\int_{0}^{1} b(s) d A_{2}(s)=\int_{0}^{1} s d s=\frac{1}{2}>0, \\
& 1-\mu_{1} \mu_{2} k_{1} k_{2}=\frac{27}{28}>0 .
\end{aligned}
$$

Condition $\left(\mathbf{H}_{0}\right)$ holds. Through calculation, $L_{1}=L_{2}=2.43299, l_{1}=l_{2}=6.80274, \omega=$ 0.01953. Choose

$$
\begin{aligned}
& f_{1}(t, x, y)=10^{-5}\left(x^{2}+y^{2}\right) \cos t+350 \sin x, \\
& f_{2}(t, x, y)=10^{-4} t\left(x^{2}+y^{2}\right)+350 \sin y, \\
& f_{10}=350>348.32258=\varphi_{p_{1}}\left(\frac{l_{1}}{\omega}\right), \\
& f_{20}=350>348.32258=\varphi_{p_{2}}\left(\frac{l_{2}}{\omega}\right) \\
& f_{1 \infty}=+\infty>348.32258=\varphi_{p_{1}}\left(\frac{l_{1}}{\omega}\right), \\
& f_{2 \infty}=+\infty>348.32258=\varphi_{p_{2}}\left(\frac{l_{2}}{\omega}\right) .
\end{aligned}
$$


Take $d_{1}=d_{2}=2, r_{1}=180$, we have

$$
\begin{aligned}
& f_{1}(t, x, y) \leq 350.648<360=d_{1} r_{1}, \\
& f_{2}(t, x, y) \leq 356.48<360=d_{2} r_{1}, \quad 0 \leq t \leq 1,0 \leq x, y \leq 180 .
\end{aligned}
$$

Then, by Theorem 3.1, system (4.1) has at least two positive solutions $\left(u_{1}, v_{1}\right)$ and $\left(u_{2}, v_{2}\right)$ such that $0<\left\|\left(u_{1}, v_{1}\right)\right\|<180<\left\|\left(u_{2}, v_{2}\right)\right\|$.

\section{Acknowledgements}

The author thanks the anonymous reviewers for carefully reading this paper and constructive comments.

\section{Funding}

This work is supported by the National Natural Science Foundation of China $(11701252,11671185,61703194)$, the Science Research Foundation for Doctoral Authorities of Linyi University (LYDX2016BS080), the Natural Science Foundation of Shandong Province of China (ZR2018MA016), and the Applied Mathematics Enhancement Program of Linyi University.

\section{Availability of data and materials}

Not applicable.

\section{Competing interests}

The author declares that she has no competing interests.

\section{Authors' contributions}

This entire work has been completed by the author. The author read and approved the final manuscript.

\section{Publisher's Note}

Springer Nature remains neutral with regard to jurisdictional claims in published maps and institutional affiliations.

\section{Received: 19 March 2019 Accepted: 21 August 2019 Published online: 02 September 2019}

\section{References}

1. Arafa, A.A.M., Rida, S.Z., Khalil, M.: Fractional modeling dynamics of HIV and CD4 ${ }^{+}$T-cells during primary infection. EPJ Nonlinear Biomed. Phys. 2012, 6 (2012)

2. Wang, G., Agarwal, R., Cabada, A.: Existence results and monotone iterative technique for systems of nonlinear fractional differential equations. Appl. Math. Lett. 25(6), 1019-1024 (2012)

3. Wang, Y., Liu, L., Zhang, X., Wu, Y.: Positive solutions of a fractional semipositone differential system arising from the study of HIV infection models. Appl. Math. Comput. 258, 312-324 (2015)

4. Cui, Y.: Uniqueness of solution for boundary value problems for fractional differential equations. Appl. Math. Lett. 51, 48-54 (2016)

5. Wang, Y.: Positive solutions for fractional differential equation involving the Riemann-Stieltjes integral conditions with two parameters. J. Nonlinear Sci. Appl. 9, 5733-5740 (2016)

6. Zou, Y., He, G.: On the uniqueness of solutions for a class of fractional differential equations. Appl. Math. Lett. 74 68-73 (2017)

7. Zhu, B., Liu, L., Wu, Y.: Local and global existence of mild solutions for a class of semilinear fractional integro-differential equations. Fract. Calc. Appl. Anal. 20(6), 1338-1355 (2017)

8. Wang, Y., Liu, L.: Uniqueness and existence of positive solutions for the fractional integro-differential equation. Bound. Value Probl. 2017, 12 (2017)

9. Cui, Y., Ma, W., Wang, X., Su, X.: Uniqueness theorem of differential system with coupled integral boundary conditions. Electron. J. Qual. Theory Differ. Equ. 9, 1-10 (2018)

10. Zhang, X., Liu, L., Wu, Y., Zou, Y.: Existence and uniqueness of solutions for systems of fractional differential equations with Riemann-Stieltjes integral boundary condition. Adv. Differ. Equ. 2018, 204 (2018)

11. Jiang, J., Liu, W., Wang, H.: Positive solutions to singular Dirichlet-type boundary value problems of nonlinear fractional differential equations. Adv. Differ. Equ. 2018, 169 (2018)

12. Cui, Y., Ma, W., Sun, Q., Su, X.: New uniqueness results for boundary value problem of fractional differential equation. Nonlinear Anal. 23(1), 31-39 (2018)

13. Hao, X., Zhang, L., Liu, L.: Positive solutions of higher order fractional integral boundary value problem with a parameter. Nonlinear Anal., Model. Control 24(2), 210-223 (2019)

14. Zhang, X., Jiang, J., Wu, Y., Cui, Y.: Existence and asymptotic properties of solutions for a nonlinear Schrödinger elliptic equation from geophysical fluid flows. Appl. Math. Lett. 90, 229-237 (2019)

15. Yue, Y., Tian, Y., Bai, Z.: Infinitely many nonnegative solutions for a fractional differential inclusion with oscillatory potential. Appl. Math. Lett. 88, 64-72 (2019)

16. Dumitru, B., Asef, M., Shahram, R.: On the existence of solutions for some infinite coefficient-symmetric Caputo-Fabrizio fractional integro-differential equations. Bound. Value Probl. 2017, 145 (2017)

17. Denton, Z., Ramírez, J.D.: Existence of minimal and maximal solutions to RL fractional integro-differential initial value problems. Opusc. Math. 37(5), 705-724 (2017) 
18. Lyons, J.W., Neugebauer, J.T.: Positive solutions of a singular fractional boundary value problem with a fractional boundary condition. Opusc. Math. 37(3), 421-434 (2017)

19. Ambrosio, V:: Zero mass case for a fractional Berestycki-Lions-type problem. Adv. Nonlinear Anal. 7(3), 365-374 (2018)

20. Zhou, Z., Qiao, Y.: Solutions for a class of fractional Langevin equations with integral and anti-periodic boundary conditions. Bound. Value Probl. 2018, 152 (2018)

21. Giovanni, M.B., Radulescu, V.D., Servadei, R.: Variational Methods for Nonlocal Fractional Problems. Encyclopedia of Mathematics and Its Applications, vol. 162. Cambridge University Press, Cambridge (2016)

22. Papageorgiou, N.S., Radulescu, V.D., Repovs, D.D.: Nonlinear Analysis-Theory and Methods. Springer Monographs in Mathematics. Springer, Cham (2019)

23. Cabada, A., Wang, G.: Positive solutions of nonlinear fractional differential equations with integral boundary value conditions. J. Math. Anal. Appl. 389, 403-411 (2012)

24. Cabada, A., Wang, G.: Nonlinear fractional differential equations with integral boundary value conditions. Appl. Math. Comput. 228, 251-257 (2014)

25. Ding, Y., Wei, Z., Xu, J., O'Regan, D.: Extremal solutions for nonlinear fractional boundary value problems with p-Laplacian. J. Comput. Appl. Math. 288, 151-158 (2015)

26. Zhang, X., Liu, L., Wu, Y., Cui, Y.: Entire blow-up solutions for a quasilinear $p$-Laplacian Schrodinger equation with a nonsquare diffusion term. Appl. Math. Lett. 74, 85-93 (2017)

27. Bai, C.: Existence and uniqueness of solutions for fractional boundary value problems with $p$-Laplacian operator. Adv. Differ. Equ. 2018, 4 (2018)

28. Wu, J., Zhang, X., Liu, L., Wu, Y., Cui, Y.: The convergence analysis and error estimation for unique solution of a p-Laplacian fractional differential equation with singular decreasing nonlinearity. Bound. Value Probl. 2018, 82 (2018)

29. Wang, Y.: Existence and nonexistence of positive solutions for mixed fractional boundary value problem with parameter and p-Laplacian operator. J. Funct. Spaces 2018, Article ID 1462825 (2018)

30. Guo, L., Liu, L., Wu, Y.: Iterative unique positive solutions for singular $p$-Laplacian fractional differential equation system with several parameters. Nonlinear Anal., Model. Control 23(2), 182-203 (2018)

31. Guo, L., Liu, L.: Maximal and minimal iterative positive solutions for singular infinite-point $p$-Laplacian fractional differential equations. Nonlinear Anal., Model. Control 23(6), 851-865 (2018)

32. Li, A., Wei, C.: On fractional p-Laplacian problems with local conditions. Adv. Nonlinear Anal. 7(4), 485-496 (2018)

33. Wu, J., Zhang, X., Liu, L., Wu, Y.: Twin iterative solutions for a fractional differential turbulent flow model. Bound. Value Probl. 2016, 98 (2016)

34. Li, S., Zhang, X., Wu, Y., Caccetta, L.: Extremal solutions for $p$-Laplacian differential systems via iterative computation. Appl. Math. Lett. 26, 1151-1158 (2013)

35. Zhang, X., Liu, L., Wiwatanapataphee, B., Wu, Y.: The eigenvalue for a class of singular $p$-Laplacian fractional differential equations involving the Riemann-Stieltjes integral boundary condition. Appl. Math. Comput. 235, 412-422 (2014)

36. Wang, Y., Jiang, J.: Existence and nonexistence of positive solutions for the fractional coupled system involving generalized p-Laplacian. Adv. Differ. Equ. 2017, 337 (2017)

37. Ren, T., Li, S., Zhang, X., Liu, L.: Maximum and minimum solutions for a nonlocal $p$-Laplacian fractional differential system from eco-economical processes. Bound. Value Probl. 2017, 118 (2017)

38. Liu, X., Jia, M., Ge, W.: The method of lower and upper solutions for mixed fractional four-point boundary value problem with p-Laplacian operator. Appl. Math. Lett. 65, 56-62 (2017)

39. Hao, X., Wang, H., Liu, L., Cui, Y.: Positive solutions for a system of nonlinear fractional nonlocal boundary value problems with parameters and p-Laplacian operator. Bound. Value Probl. 2017, 182 (2017)

40. Yan, F., Zuo, M., Hao, X.: Positive solution for a fractional singular boundary value problem with $p$-Laplacian operator. Bound. Value Probl. 2018, 51 (2018)

41. Miller, K.S., Ross, B.: An Introduction to the Fractional Calculus and Fractional Differential Equations. Wiley, New York (1993)

42. Podlubny, l.: Fractional Differential Equations. Mathematics in Science and Engineering, vol. 198. Academic Press, New York (1999)

43. $\mathrm{Xu}, \mathrm{J}$., Dong, W.: Existence and uniqueness of positive solutions for a fractional boundary value problem with p-Laplacian operator. Acta Math. Sinica (Chin. Ser.) 59, 385-396 (2016)

44. Zi, Y., Wang, Y.: Positive solutions for Caputo fractional differential system with coupled boundary conditions. Adv. Differ. Equ. 2019, 80 (2019)

45. Guo, D., Lakshmikantham, V:: Nonlinear Problems in Abstract Cones. Academic Press, New York (1988)

\section{Submit your manuscript to a SpringerOpen ${ }^{\circ}$ journal and benefit from:}

- Convenient online submission

- Rigorous peer review

- Open access: articles freely available online

- High visibility within the field

- Retaining the copyright to your article

Submit your next manuscript at $\gg$ springeropen.com 